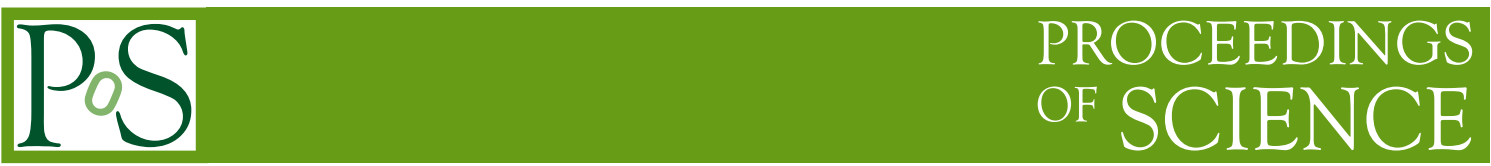

\title{
The Tracking System of HERD
}

\section{Perrina ${ }^{* a}$, G. Ambrosi ${ }^{b}$, P. Azzarello ${ }^{a}$, F. Cadoux ${ }^{a}$, E. Catanzani ${ }^{b, c}$, Y. Favre $^{a}$, D. La Marra $^{a}$, G. Silvestre ${ }^{b, c}$, J. Wang ${ }^{a, d}$ and X. Wu ${ }^{a}$ for the HERD Collaboration ${ }^{\dagger}$}

${ }^{a} \mathrm{DPNC}$, Université de Genève, $\mathrm{CH}-1211$ Genève 4, Switzerland

${ }^{b}$ INFN Sezione di Perugia, I-06100 Perugia, Italy

${ }^{c}$ Università di Perugia, I-06100 Perugia, Italy

${ }^{d}$ Institute of High Energy Physics (IHEP), Chinese Academy of Sciences, Beijing 100049, China

E-mail: chiara.perrina@unige.ch

The High Energy cosmic-Radiation Detection (HERD) facility will be one of the future astronomy missions on board the Chinese Space Station. The main objective of HERD is the direct detection of cosmic rays towards the "knee" region $(\sim 1 \mathrm{PeV})$, with an excellent energy resolution ( $<1 \%$ for electrons and photons at $200 \mathrm{GeV}$ and $20 \%$ for nuclei from $100 \mathrm{GeV}$ to $\mathrm{PeV}$ ), an unprecedented acceptance $\left(>1 \mathrm{~m}^{2} \mathrm{sr}\right.$ ), and long life-time ( $>10$ years). The tracking system of HERD will provide a full coverage of the 5-side sensitive HERD detector, allowing for a subdegree angular resolution and multiple redundant and independent measurements of the charge of nuclei. Both silicon strip and scintillating fiber tracking detectors are being considered. The HERD tracker design, the results of on-ground tests carried out with particle beams at CERN and comparisons with Monte Carlo simulations will be presented in this contribution.

36th International Cosmic Ray Conference -ICRC2019-

July 24th - August 1st, 2019

Madison, WI, U.S.A.

\footnotetext{
* Speaker.

${ }^{\dagger}$ for collaboration list see PoS(ICRC2019)1177
} 


\section{Introduction}

Where and how the cosmic particles are produced and accelerated? In which way do they interact and propagate before reaching the Earth? What kind of particles/nuclei are present in cosmic ray fluxes and what is their energy? Can the still elusive dark matter produce the anomalies observed in the cosmic ray spectrum at high energy ([1], [2])? The HERD mission, where HERD is the acronym of High Energy cosmic-Radiation Detection, will boost the resolution of these enigmas, for which we have not yet an answer since the discovery of cosmic particles. The HERD facility will be a flagship scientific experiment on board the Chinese Space Station (CSS). The CSS will be constructed in 2020-2022, and the HERD apparatus is expected to be launched soon after, in 2025, and to be operational for at least 10 years.

HERD aims to the search for annihilation and decay products of dark matter, both in the energy spectrum and anisotropy of electrons from $10 \mathrm{GeV}$ to $100 \mathrm{TeV}$ and in the gamma-ray spectrum from $500 \mathrm{MeV}$ to $100 \mathrm{TeV}$, and to the measurement of the energy spectrum and composition of cosmic rays (protons and nuclei up to iron) from $30 \mathrm{GeV}$ to a few $\mathrm{PeV}$. The latter will be fundamental for the understanding of stars and galaxies formation and evolution and of the extreme energetic phenomena (supernova explosions, pulsar jets, neutron star and black hole merging) in the universe. Furthermore, HERD will provide a wide field of view monitoring of the $\gamma$-ray sky from $500 \mathrm{MeV}$ that will allow the study of gamma-ray bursts, active galactic nuclei and galactic microquasars.

The main requirements for HERD in terms of energy range, of energy, angular and charge resolution, of electron/proton separation and of geometric factors are listed in Table 1. It is important to notice that the geometric factors are $\sim 10$ times the geometric factors of present detectors, with a field of view of $70^{\circ}$ off the zenith. Fig. 1 shows the exploded view of the HERD detector. From inside to outside, HERD consists of a calorimeter (CALO) for the measurement of the energy of traversing particles and for the electron/proton discrimination, a 5-side particle tracking system which will serve to reconstruct the particle trajectories and to identify the nuclei charge, a plastic scintillator detector (PSD) for the measurement of low energy gamma-rays and for a redundant measurement of the nuclei charge, and a transition radiation detector (TRD) used for the energy calibration. Two possible tracker options are under study: a 5-side silicon strip tracker (STK) and a 4-side scintillating fiber tracker (FIT) with a silicon strip tracker on the top. The second option is depicted in Fig. 1.

\section{The tracking System}

\subsection{STK}

The silicon tracker (STK) is designed to reconstruct the track and the absolute value of the charge of elementary particles and of nuclei with atomic number $Z \leq 26$. The top STK (Fig. 2, panel 2) consists in six x-y tracking planes of silicon strip detectors (SSDs), each plane has an active area of $133 \mathrm{~cm} \times 133 \mathrm{~cm}$ and is equipped with 28 Type A ladders, each assembled with 7 single-sided SSDs. Ladders on $\mathrm{x}$ tracking planes are placed orthogonally to ladders on y tracking planes in order to measure the $\mathrm{x}$ and $\mathrm{y}$ coordinates of the traversing particle. The lateral STKs consist in three $\mathrm{z}-\mathrm{x}$ or $\mathrm{z}-\mathrm{y}$ tracking planes of silicon strip detectors. Each plane has an active area of $95 \mathrm{~cm} \times 66.5 \mathrm{~cm}$ and is equipped with 14 Type B ladders (Fig. 2, panel 3) or 10 Type A ones (Fig. 


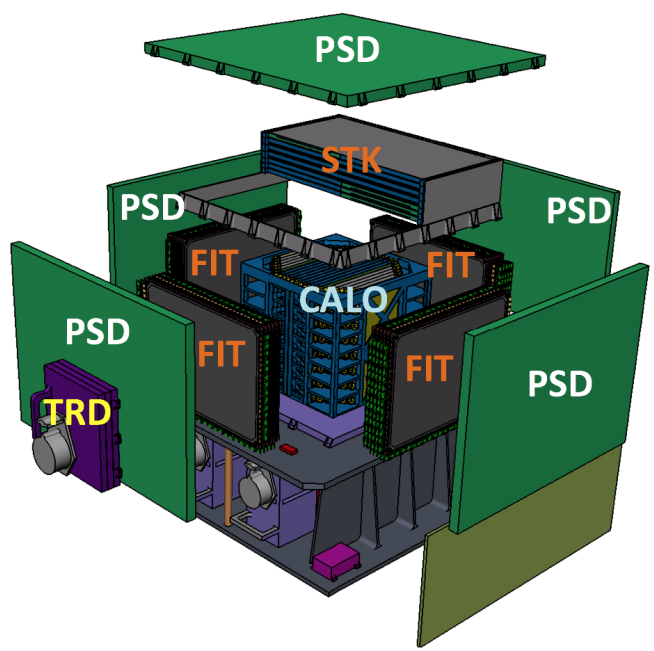

Figure 1: Exploded view of the HERD detector. The whole apparatus will have a weight $<4 \mathrm{t}$ and a volume of $(2.3 \times 2.3 \times 2.6) \mathrm{m}^{3}$.

\begin{tabular}{lc}
\hline Energy range (e/ $\gamma$ ) & $10 \mathrm{GeV}-100 \mathrm{TeV}$ \\
\hline Low energy range for $\gamma$ & $500 \mathrm{MeV}-10 \mathrm{GeV}$ \\
Energy range (nuclei) & $30 \mathrm{GeV}-3 \mathrm{PeV}$ \\
Angular resolution $(\mathrm{e} / \gamma)$ & $0.1^{\circ}$ at $10 \mathrm{GeV}$ \\
Charge resolution (nuclei) & $10 \%-15 \%$ for $Z=1-26$ \\
\hline Energy resolution (e/ $/$ ) & $<1 \%$ at $200 \mathrm{GeV}$ \\
Energy resolution (nuclei) & $20 \%$ at $100 \mathrm{GeV}-\mathrm{PeV}$ \\
\hline e/p separation power & $>10^{5}$ \\
\hline Geometric factor (e) & $>3 \mathrm{~m}^{2}$ sr at $200 \mathrm{GeV}$ \\
\hline Geometric factor (nuclei) & $>2 \mathrm{~m}^{2}$ sr at $100 \mathrm{GeV}$ \\
\hline
\end{tabular}

Table 1: Main requirements for the HERD payload

2, panel 4). Each Type B ladder is assembled with 5 single-sided SSDs. The material between the active parts will be minimized to ensure the physics performance while keeping the stability of the overall mechanics. The SSD is the elementary element of the STK and the HERD baseline is to use the same SSD used in the STK of DAMPE [3] (Fig. 2, panel 1). Thanks to $121 \mu \mathrm{m}$ of strip pitch and $242 \mu \mathrm{m}$ of read-out pitch, after a proper alignment, we expect a spatial resolution of $\sim 40$ $\mu \mathrm{m}$. The read-out of one every other strip (corresponding to 384 channels per ladder) is a balance among a good performance in terms of spatial resolution and a limited power consumption.

\subsection{FIT}

The requirement for a full coverage on the lateral sides of HERD puts limits to the location of the front-end and read-out electronics. Therefore, a scintillating fiber tracking detector represents an interesting alternative to a silicon strip tracker, thanks to fibers that can be easily adapted to the demanded geometry. There are several motivations to consider a scintillating fiber tracker: it would be simpler to build such a long detector $(>1 \mathrm{~m}$ ), the dead area would be reduced (no gap 


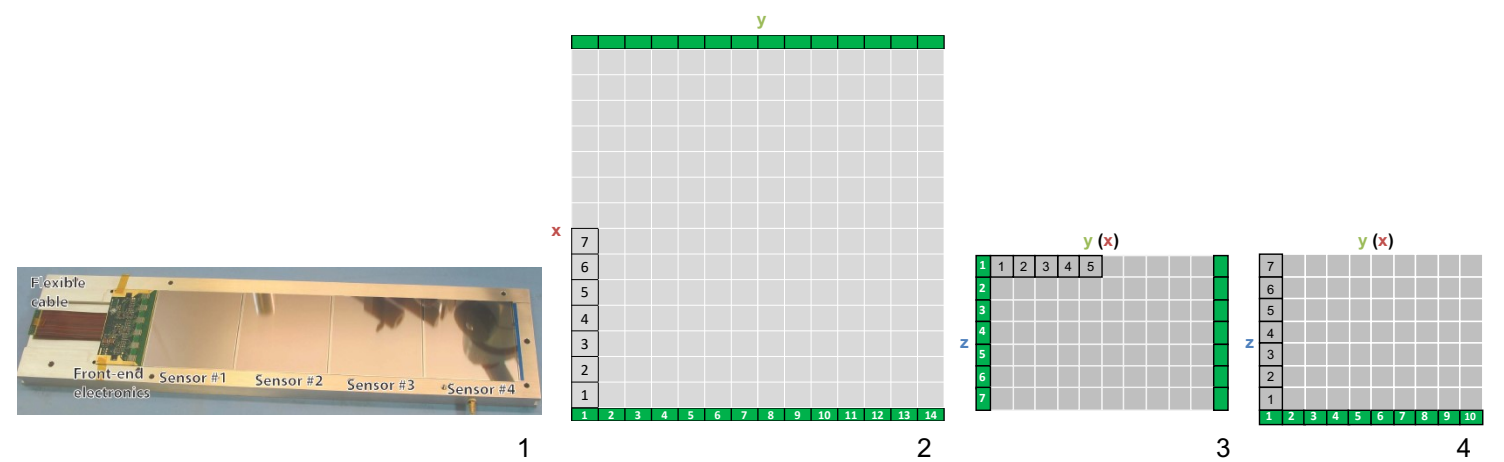

Figure 2: DAMPE ladder equipped with 4 SSDs made by Hamamatsu with an area of $95 \mathrm{~mm} \times 95 \mathrm{~mm}(1)$. Sketch of a top STK plane equipped with 28 Type A ladders (2). Sketch of a lateral STK plane equipped with 14 Type B ladders (3). Sketch of a lateral STK plane equipped with 10 Type A ladders (4).

between sensors, no dead area on the silicon detector, front-end and read-out electronics placed outside the support trays), it would be less expensive to add more layers, thus improving the point spread function of low energy photons, and more robust since wire bonds are not needed.

\subsubsection{Mechanical and electronic design}

According to the current design, the FIT consists in four identical sectors (Fig. 3 top, left). Each sector hosts $8 \mathrm{x}$-y tracking planes (Fig. 3 top, right). Each $\mathrm{x}$-plane is made of $7 \mathrm{x}$-modules and each y-plane is made of $10 \mathrm{y}$-modules (Fig. 3 bottom, left). The module is the elementary unit of the FIT detector (Fig. 3 bottom, right). It includes a scintillating fiber mat and 3 silicon photomultiplier ( $\mathrm{SiPM}$ ) arrays to read out the scintillation light. The mat is made by stacking 6
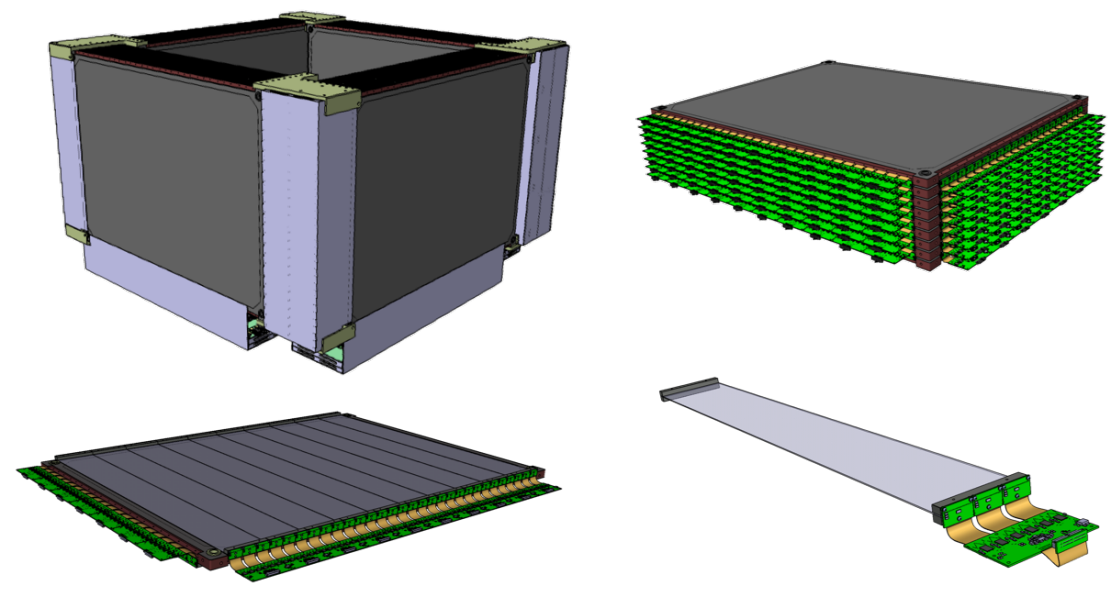

Figure 3: Sketch of the 4-side FIT detector made of 4 identical sectors (top, left); the light grey blocks (2 for each sector) encapsulate the read-out electronics. The four sectors are interconnected by eight corner feet (green in the illustration). Sketch of one sector made of 9 support trays on which $8 \mathrm{x}-\mathrm{y}$ tracking planes are placed (top, right). Sketch of one $\mathrm{x}-\mathrm{y}$ plane: the top plane is a y-plane with $10 \mathrm{y}$-modules, the $\mathrm{x}$-plane on bottom hosts $7 \mathrm{x}$-modules rotated by $90^{\circ}$ with respect to the y-modules (bottom, left). Sketch of a FIT module (bottom, right). 
layers of fibers. In Fig. 4 the cross section of a $2 \mathrm{~mm}$ mat segment is shown. The fibers are made by Kuraray (SCSF-78MJ type), they have a round section with a diameter of $250 \mu \mathrm{m}$ and a peak emission at $450 \mathrm{~nm}$ wavelength. Except for lenght and width, the FIT fiber mat is the same used for LHCb fiber tracker upgrade [4]. The only difference between a x-module and a y-module is the length of the fiber mat: $106 \mathrm{~cm}$ for the firsts and $77 \mathrm{~cm}$ for the latters. On the other side, the fiber mat width is $97.8 \mathrm{~mm}$ for both, to match 3 SiPM arrays. In such a configuration, each sector hosts 136 fiber mats and 408 SiPM arrays, therefore the whole apparatus hosts 544 fiber mats and 1632 SiPM arrays.

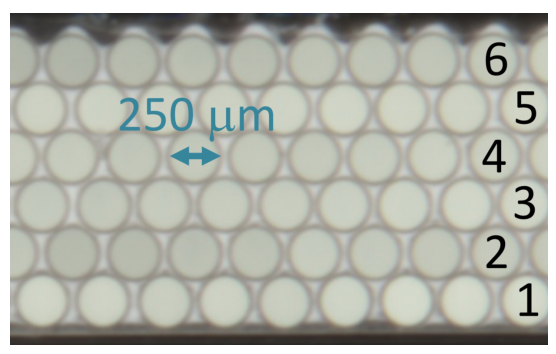

Figure 4: Cross section of a $2 \mathrm{~mm}$ width fiber mat segment. Each mat is made of 6 layers of fibers and its total width is $97.8 \mathrm{~mm}$.

Two types of SiPM array are under study: the S133552-HRQ type, made by Hamamatsu for the LHCb experiment, and the S13552-10 type, produced by Hamamatsu for the University of Geneva. The "LHCb" type has 2 chips/array and 64 channels/chip. Each channel contains $4 \times 26$ pixels of $57.5 \mu \mathrm{m} \times 62.5 \mu \mathrm{m}$ area, with a gain of $10^{6}$. A picture of the S133552-HRQ SiPM array is shown in Fig. 5. The S13552-10 type has the same footprint of the one used by LHCb, with 2 chips of 64 channels, customized with $23 \times 163$ square pixels/channel of $10 \mu \mathrm{m}$ side, with a gain of $10^{5}$. The details of the interface between the fiber mat and the SiPM arrays are shown in Fig. 6. Each SiPM array is mounted on a small printed circuit board (PCB), which is screwed on the end-piece of the FIT module. The three PCBs are connected to the main body of the front-end board (FEB) through flex cables. In addition to the three SiPM arrays, the FEB hosts the front-end circuit itself. On the back side of the SiPM PCB is mounted a PT100 thermal sensor, to allow for a thermal correction of the bias voltage. The FEB circuit reads out serially the three SiPM arrays using six VATA64 HDR 16.2 ASICs. In total, the FEB provides 384 analogical signals. The eight tracking planes are

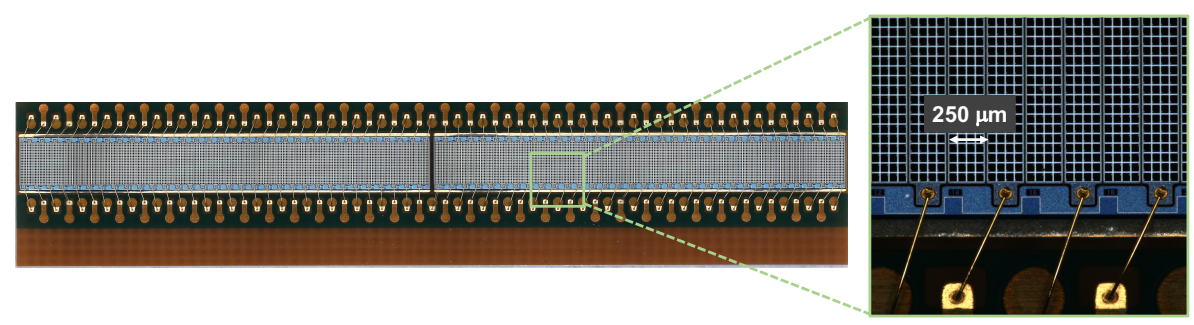

Figure 5: Picture of a S133552-HRQ ("LHCb") SiPM array. On the right, eight channels are zoomed in. The channel pitch is $250 \mu \mathrm{m}$.

glued on nine support trays. Seven trays are equipped on both sides, while the top and bottom ones are equipped only on the side facing the interior of the tracker. A support tray will be made of an 

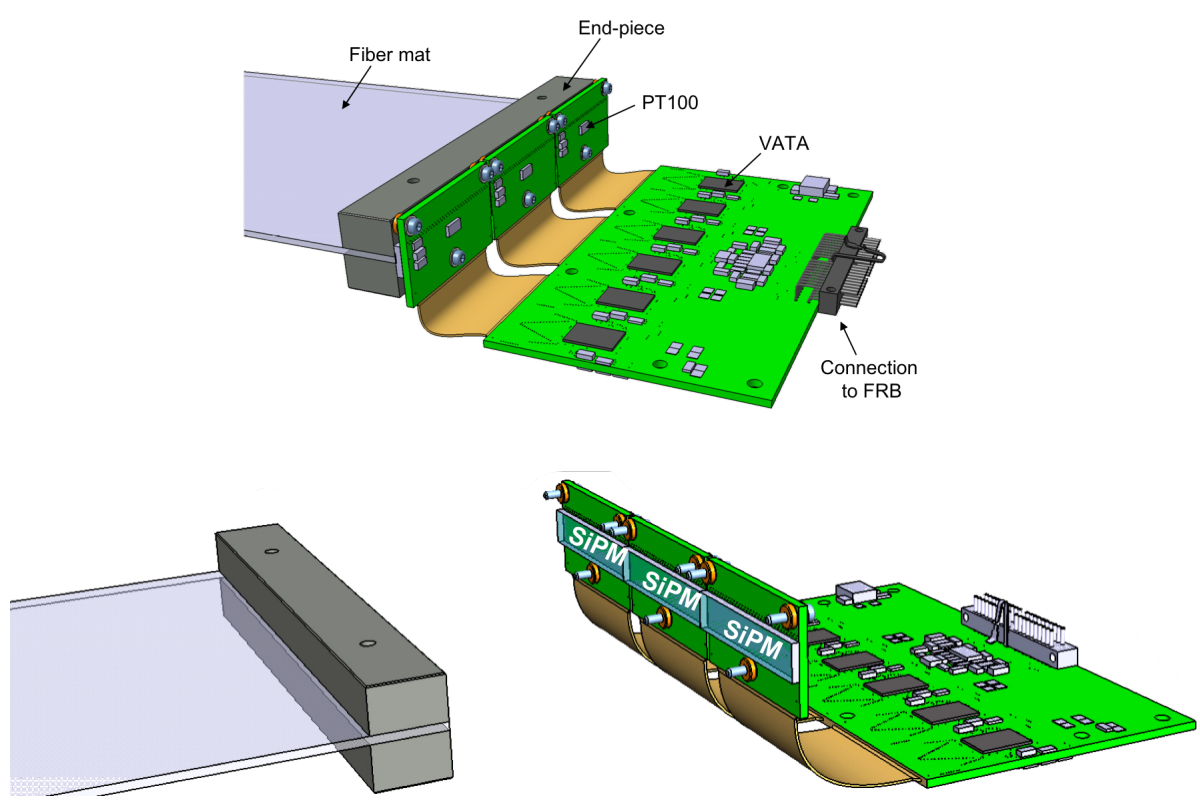

Figure 6: Sketch highlighting the end part of the FIT module. The 3 SiPM arrays are mounted on 3 PCBs connected to the main FEB via 3 flex cables. The 3 PCBs are screwed on the end-piece. The position of the 3 PT100 thermal sensors, the 6 VATAs and the connector to the FIT read-out board is shown.

AIREX ${ }^{\circledR}$ foam sandwiched between two face sheets of carbon fiber reinforced polymer (CFRP). The possibility to use a Nomex ${ }^{\circledR}$ honeycomb structure instead of the AIREX® foam is under study. With such a structure, the tray is light but rigid enough to sustain the vibrations which occur during a rocket launch. In the current design study, a tray is $20 \mathrm{~mm}$ thick, the distance between two tracking planes is $25 \mathrm{~mm}$, therefore the FIT envelope is $\sim(1.5 \times 1.5 \times 1) \mathrm{m}^{3}$, for an overall mass of $\sim 250 \mathrm{~kg}$.

The data acquisition circuit necessary to read out the FIT modules is named FRB (FIT readout board). This circuit is developed over two boards: the first one is dedicated to the power supply of the electronics and the FIT modules, the second one manages the digital control, the communication with the main DAQ system and the FEB read-out. Each FRB is designed to read out 16 FIT modules. Therefore, nine FRBs will be necessary to read out a FIT sector: four will be mounted on its short edge, and five on its long edge. Two groups of eight FIT modules will be connected to the FRB, through two flex cables, one for each group. The prototype version will include an ADC circuit. This is due to the ASICs used for the prototype front-end, which do not include an ADC. The final front-end design foresees an ASIC with an integrated ADC, this is the reason why the FRB does not host ADCs. A FIT sector provides 52224 channels and the entire FIT 208896 channels; depending on the capability of the DAQ main system of HERD, a data compression algorithm will be implemented in the FRB.

\subsubsection{Beam test analysis results}

Different fiber module prototypes have been assembled at the University of Geneva, and tested during several beam test campaigns. Three prototypes, made of a $77 \mathrm{~cm}$ length fiber mat, of which two coupled with a "LHCb" and one with a "10 $\mu \mathrm{m}$ " SiPM array have been under study at the beam 
test held in October 2018 at the North Area of the SPS at CERN. A preliminary version of the FEB, with two VATA ASICs, reading out one SiPM array, has been used. Preliminary results have been obtained by analyzing the data collected with the $400 \mathrm{GeV} / \mathrm{c}$ proton beam hitting orthogonally the center of the mat ( $~ 38.5 \mathrm{~cm}$ from the SiPM array) with the "LHCb" and the "10 $\mu \mathrm{m}$ " SiPM arrays powered with an over-voltage of $2 \mathrm{~V}$ and $6.5 \mathrm{~V}$ respectively. In Fig. 7, the hit residual distributions, fitted with a double gaussian, are displayed. They reveal a good spatial resolution: $53.9 \mu \mathrm{m}$ and $59.1 \mu \mathrm{m}$ for "LHCb" and "10 $\mu \mathrm{m}$ " SiPM arrays respectively. $70 \mu \mathrm{m}$ would be sufficient for our application because it guarantees an angular resolution of $0.1^{\circ}$. The hit efficiency is about $99.4 \%$.
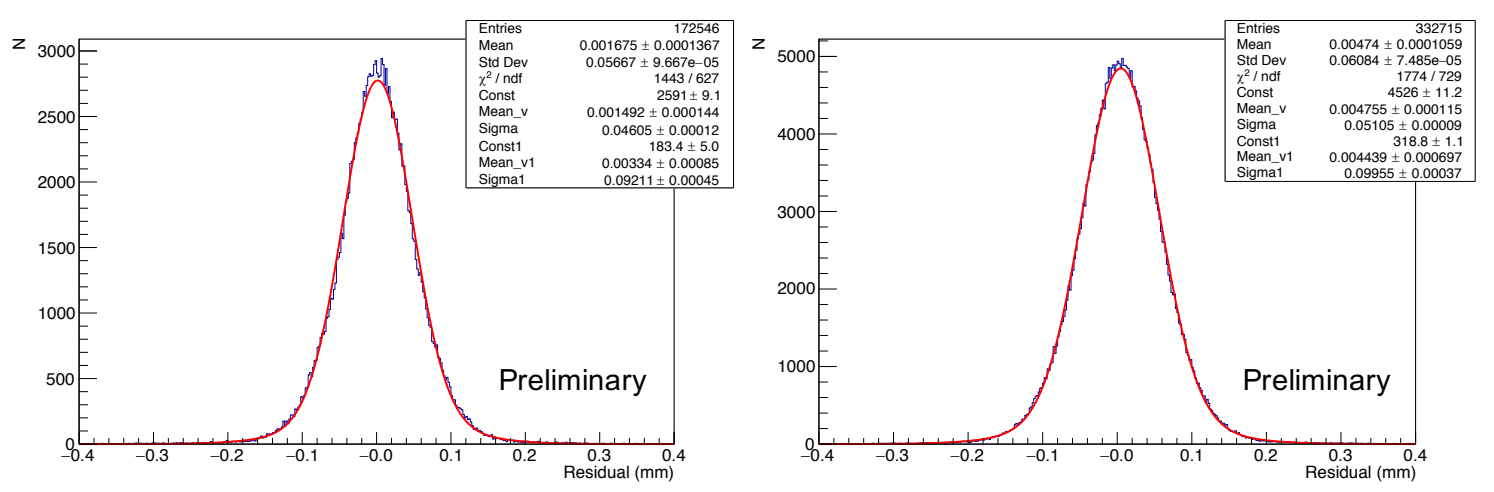

Figure 7: Beam test results for two FIT prototype modules made of $77 \mathrm{~cm}$ length fiber mat read out by a "LHCb" (left) and a "10 $\mu$ m" (right) SiPM array. The hit residual distribution fitted with a double Gaussian is shown.

To study the performances of the FIT detector and to optimise its geometry, a dedicated Monte Carlo simulation is being developed, based on the Geant4 framework. In Fig. 8 the distributions of the cluster size (left) and of the cluster charge (right) for Monte Carlo (blue) and beam test (red) events read out by the " $10 \mu \mathrm{m}$ " SiPM arrays are shown. The Monte Carlo simulations confirm the most probable cluster size being 2 channels, while 11.5 p.e. is the mean cluster light yield.
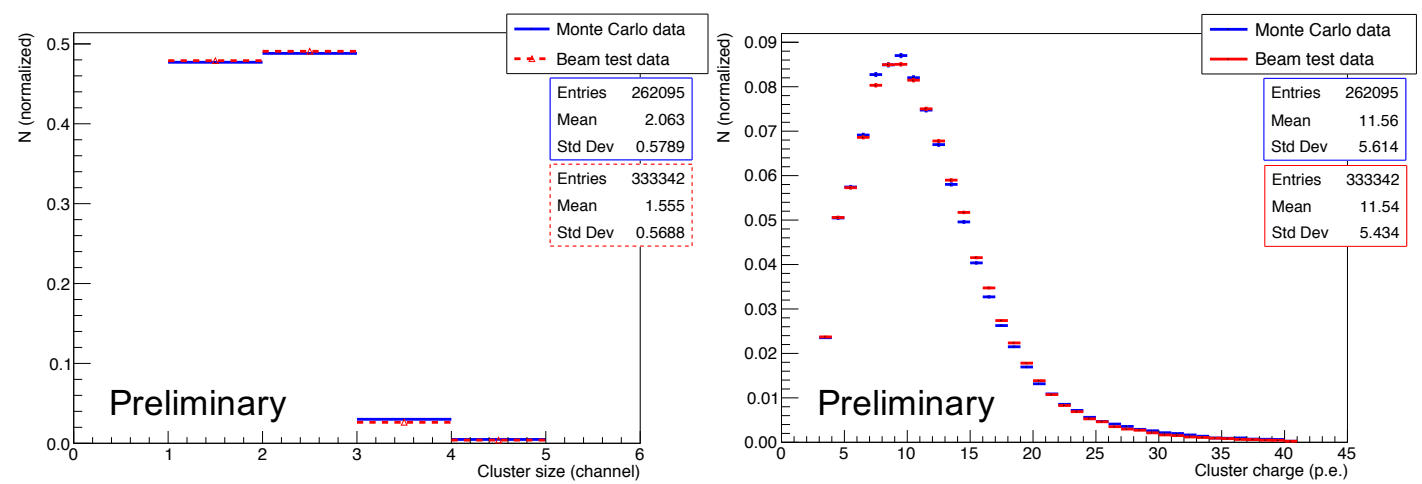

Figure 8: Distribution of the size (left) and of the charge (right) of clusters for the reconstructed Monte Carlo (blue) and beam test (red) track events. 


\section{Conclusions}

HERD will be taking data on board the CSS from 2025 for more than 10 years. It will be a calorimetric detector with unprecedented acceptance. HERD will play a fundamental role in cosmic ray physics, dark matter search and gamma-ray astronomy. It will be the only space-borne high energy gamma-ray detector, after the conclusion of the Fermi mission. Several studies and tests for further improvements and optimization are ongoing.

\section{Acknowledgement}

The authors thank the colleagues of the EPFL LHCb group for the fiber procurement and their significant support.

\section{References}

[1] M. Aguilar et al. (AMS Collaboration), Electron and Positron Fluxes in Primary Cosmic Rays Measured with the Alpha Magnetic Spectrometer on the International Space Station, Phys. Rev. Lett. 113 (2014) 221102.

[2] G. Ambrosi et al. (DAMPE Collaboration), Direct detection of a break in the teraelectronvolt cosmic-ray spectrum of electrons and positrons, Nature 552 (2017) 63-66 [astro-ph.HE/1711.10981].

[3] A. Tykhonov et al. (DAMPE Collaboration), Internal alignment and position resolution of the silicon tracker of DAMPE determined with orbit data, Nucl. Instrum. Meth. A893 (2018) 43-56 [physics.ins-det/1712.02739].

[4] P. Hopchev (for the LHCb SciFi Tracker Collaboration), SciFi: A large Scintillating Fibre Tracker for $L H C b$, [physics.ins-det/1710.08325]. 